\title{
Autocontrol y consumo de drogas
}

\author{
Francisca lópez-Torrecillas*, Isabel Peralta*, Marina J. Muñoz-Rivas**, Juan F. Godoy* \\ *Departamento de Personalidad, Evaluación y Tratamiento Psicológico. Facultad de Psicología. Universidad de Granada. \\ **Departmento de Psicología Biológica y de la Salud. Facultad de Psicología. Universidad Autonoma de Madrid.

\section{RESUMEN}

En este estudio se intenta relacionar el consumo de drogas con el autocontrol, estilo atribucional, asertividad y autoeficacia. Participaron en el estudio 53 sujetos consumidores de alcohol, hachís, cocaína y heroína que fueron reclutados en lugares de encuentro de jóvenes (gimnasios, asociaciones, bares y centros de estudios y trabajo). Las medidas que se utilizaron fueron el Cuestionario de Autocontrol de Rosenbaum (1980), el Cuestionario de Estilo Atribucional de Peterson et al. (1982), el Inventario de Asertividad de Rathus (1973) y la Escala de Autoeficacia de Sherer et al. (1982). Se realizaron dos análisis de regresión lineal múltiple para cada una de las variables que definen el consumo de drogas: cantidad de droga consumida (alcohol, hachís, cocaína y heroína) y cronicidad del consumo de drogas (años de consumo de alcohol, hachís, cocaína y heroína). En los dos análisis de regresión lineal múltiple las variables independientes son las mismas variables de personalidad (estilo atribucional para los éxitos y estilo atribucional para los fracasos, asertividad, autocontrol y autoeficacia) y las variables dependientes son las puntuaciones estandarizadas de la cantidad de consumo de droga y de la cronicidad del consumo de drogas. Los resultados muestran que el autocontrol y la asertividad están relacionados con el consumo de drogas (cantidad y cronicidad del consumo). La relación entre el autocontrol y el consumo de drogas es inversa, mientras que la relación entre la asertividad y el consumo de drogas es directa. Nuestros resultados ponen de manifiesto la conveniencia de intervenir en las variables mencionadas como forma de prevención en el consumo posterior.

Palabras clave: autocontrol, asertividad, consumo de drogas.

\section{ABSTRACT}

In this study, we attempted to relate drug use with self-control, attributional style, assertiveness and self-efficacy. 53 subjects, alcohol, hashish, cocaine and heroin users, took part in the study. They were contacted in youth hang-outs such as sports clubs, bars, associations, and study or work centres. The measurement tools used in the study were: Self-control Questionnaire by Rosenbaum (1980), Attributional Style Questionnaire by Peterson et al. (1982), Assertiveness Inventory by Rathus (1973) and Self-efficacy Scale by Sherer et al. (1982). Two lineal multiple regression analyses were carried out, for each of the variables that make up the severity of the use, these variables being: quantity used (alcohol, hashish, cocaine and heroin) and drug use chronicity (years using alcohol, hashish, cocaine and heroin). In the two multiple lineal regression analyses, the independent variables are the same personality variables (self-control, assertiveness, self-efficacy, attributional style for success, attributional style for failure) and the dependent variables are standardized scores for the quantity of drug use and drug use chronicity. Results show that self-control and assertiveness are related to drug use (quantity and chronicity). The relation between self-control and drug use is inverse whereas the relation between assertiveness and drug use is a direct one. Our results highlight the need to intervene in the variables examined as a way of preventing or reducing substance abuse.

Key words: self-control, assertiveness, drug use.

\section{1.- INTRODUCCIÓN}

A unque son todavía escasos los estudios que relacionan variables de personalidad con las drogodependencias, cabe deducir que en aquellas situaciones en las que el sujeto se ve inmer- so por la presión para consumir drogas y tiene que exhibir una determinada estrategia de afrontamiento, están mediando variables tales como autocontrol, asertividad, estilo atribucional y autoeficacia. El estudio de las relaciones entre estas variables permitirá diseñar intervenciones eficaces tanto de prevención como de tratamiento. 


\section{1.- Autocontrol.}

En la línea que acabamos de mencionar, el autocontrol resulta de vital importancia en la fase de mantenimiento del consumo de drogas, pues hace que el individuo sea capaz de mantener un consumo moderado. Por el contrario, su ausencia conlleva un nivel de consumo excesivo (D’Elio et al., 1996; Kahler et al., 1995; Room, 1989; Santacreu y Froján, 1992; Sterling et al., 1996; Shiffman y Wills, 1985; Wills, 1994).

En el estudio de Santacreu y Froján (1992) se miden una serie de variables, entre las que cabe destacar el autocontrol, conductas divergentes a la norma, nivel de ansiedad, estrés, etc. En muestras de sujetos de edades comprendidas entre 11 y 22 años, donde el primer grupo consume alcohol y tabaco, el segundo, hachís, pegamento y somníferos y el tercero, heroína, cocaína y anfetaminas. Los resultados confirman que el tercer grupo presenta niveles más bajos de autocontrol que el segundo y el primer grupo.

Wills (1994) estudia la relación entre autoestima, autocontrol y consumo de tabaco, alcohol y hachís, en una muestra de adolescentes estudiantes. Encuentra que la percepción de control interna está inversamente relacionada con el consumo de las drogas estudiadas.

D’Elio et al. (1996) afirman que la percepción de acontecimientos incontrolables aumenta el riesgo de consumir drogas; esta afirmación se deriva del estudio que hicieron con adolescentes de 11 años de edad que consumían alcohol, hachís y cocaína. Concluyen que la percepción de acontecimientos incontrolables predecía el consumo de drogas.

Sterling et al. (1996) estudian el control personal con relación al consumo de drogas en una muestra de consumidores de cocaína que inician tratamiento. El análisis de datos, en su caso, indica que el control personal predice la tasa de retención en el tratamiento.

\section{2.- Estilo Atribucional.}

Siguiendo con los estudios de estilo atribucional, señalamos su relevancia porque resaltan que el consumo de alcohol y drogas está relacionado con atribuciones inadecuadas, es decir, contrarias a percepciones favorables (internas y estables) (Abramson, et al., 1978; Rizley, 1978). De acuerdo con estos postulados, Dowd et al., (1986); Echeburúa y Elizondo (1988); Reich y Gutiérres (1987) destacan la influencia del estilo atribucional en drogodependientes. Específicamente, Dowd et al. (1986) analizan el estilo atribucional, autoeficacia y ansiedad en alcohólicos rehabilitados y bebedores sociales. Los resultados indican que no existen diferencias significativas entre los tres grupos en cuanto a ansiedad y autoeficacia, pero los alcohólicos presentan atribuciones internas y específicas de sus éxitos, considerándolas como acontecimientos transitorios.

En la misma línea, Echeburúa y Elizondo (1988) estudian el estilo atribucional y locus de control de una muestra de alcohólicos, en comparación con un grupo de no-alcohólicos. Destacan que los alcohólicos, en relación con los no alcohólicos poseen un estilo atribucional más externo e inestable ante los éxitos y más interno y global ante los fracasos, además de un locus de control más externo.

\section{3.- Asertividad.}

El interés de la asertividad en relación con las drogodependencias data desde los años 70. Desde esta década, existe bastante consenso en incluir entrenamiento en asertividad dentro del tratamiento de alcohólicos y drogodependientes; una de las razones que se señalan es que los individuos presentan dificultades en la interacción social y eficacia interpersonal desde la infancia. Van Hasselt et al. (1993) exploran la relación entre consumo de alcohol, drogas y asertividad en sujetos sometidos a tratamiento para su dependencia. El análisis de sus datos señala que unos sujetos se muestran más agresivos y otros menos asertivos. Para interpretar estos resultados los autores plantean el concepto de asertividad como un continuo, en el que en un extremo estaría la agresividad, en el otro la pasividad y en el punto medio la habilidad adecuada.

De otra parte, Wills et al. (1989) reflejan que el consumo de drogas está asociado con diferentes dimensiones de la asertividad. Estos autores utilizaron dimensiones referidas al desarrollo de estrategias para establecer relaciones sociales, para establecer compromisos de pareja y para rehusar al consumo de alcohol y drogas ante situaciones caracterizadas por presión externa. La muestra de estudio era estudiantes universitarios que consumían tabaco, hachís y alcohol. El análisis de datos reflejó que la asertividad general estaba directamente relacionada con el consumo de alcohol e inversamente relacionada con la dimensión de estrategias para rechazar el consumo bajo presión social.

\section{4.- Autoeficacia.}

En los estudios que relacionan la autoeficacia y el comportamiento adictivo, existe bastante consenso entre los autores (Baldwin et al., 1993; Hays y Ellickson, 1990; Rist y Watzl, 1983; Sadowski et al., 1993) en afirmar que, cuanto más bajas sean las expectativas del sujeto, más vulnerable será en el momento del inicio de consumo, en la conducta de mantenimiento y en las posibles recaídas. Rist y Watzl (1983) afirman que la autoeficacia es una variable protectora para la abstinencia del alcohol. Esta afirmación deriva 
del estudio que realizaron para comprobar la relación entre la creencia de los sujetos de ser capaces de mantenerse abstinentes y el comportamiento abstinente en sí. Para ello utilizaron una muestra de sujetos que habían realizado tratamiento para su dependencia del alcohol, habiendo transcurrido un período de tres meses desde la finalización del tratamiento. En el estudio se midió autoeficacia, asertividad y estrategias para rehusar al consumo de alcohol. Se comprobó que los sujetos que habían recaído no diferían en asertividad y en la habilidad para rehusar al consumo de alcohol, sino en la convicción de sentirse capaces de mantener la abstinencia en el momento de iniciar el tratamiento.

En la misma línea se desarrolla el estudio de Hays y Ellickson (1990). Estos autores observan que existe una relación entre la autoeficacia para rehusar al consumo de alcohol y marihuana, bajo presión de grupo, y las conductas dirigidas hacia el mantenimiento de la abstinencia. En este trabajo se mide la autoeficacia, que incluía el nivel de resistencia que el sujeto percibía de sí mismo ante la presión del grupo en una situación de laboratorio y el mismo nivel de resistencia ante la presión del grupo en situaciones reales. El estudio se realizó con una muestra de sujetos que consumían hachís y alcohol. Con los datos obtenidos elaboraron una escala de autoeficacia, encontrando que las puntuaciones altas están relacionadas con conductas de abstinencia y que la autoeficacia para resistirse al consumo de una droga bajo presión social se generaliza para otras drogas.

El estudio de Baldwin et al. (1993) corrobora la relación entre autoeficacia y comportamientos dirigidos hacia el control del consumo. Utilizan una muestra de estudiantes para medir las expectativas de los efectos del alcohol, el consumo de alcohol y la autoeficacia. Encuentran que los sujetos que presentan puntuaciones bajas en autoeficacia (referida a la estrategia para rehusar el consumo de alcohol), piensan que el alcohol les permite ser más asertivos, estar más relajados y mejorar sus relaciones sexuales, consumen alcohol más frecuentemente y en mayor cantidad que aquellos que presentan puntuaciones más altas en autoeficacia y no piensan que el alcohol mejore sus relaciones sexuales y sociales ni les relaje. Estos últimos tienden a mantener un nivel bajo de consumo de alcohol en frecuencia y cantidad.

Por último, algunos autores (Sadowski et al. 1993) piensan que el tratamiento dirigido hacia la mejora de las expectativas de eficacia, permite aumentar la autoeficacia de los drogodependientes y, consecuentemente, el mantenimiento de la abstinencia. Su idea se basa en los resultados de un estudio con estudiantes, que habían realizado tratamiento para su dependencia al alcohol, cocaína, hachís y otros medicamentos ingeridos sin prescripción médica. Se comparan los datos con los de la muestra que consumía las mismas dro- gas pero nunca había realizado tratamiento. Los resultados indicaron que los sujetos que habían realizado tratamiento presentaron puntuaciones más altas en autoeficacia que aquéllos que no habían recibido tratamiento.

\section{2.- OBJETIVOS.}

Hemos de indicar que, salvando las distancias que establece, en ocasiones, el uso de medidas no convergentes para el registro de las variables relevantes al problema, que no siempre es suficiente, los autores que trabajan en este campo coinciden en señalar, que niveles bajos de autocontrol y de autoeficacia, un estilo atribucional interno y específico o externo e inestable para los éxitos, niveles altos de agresividad o bajos de asertividad y falta de estrategias para rehusar al consumo de drogas, están relacionadas con las drogodependencias. Sin embargo, para analizar de manera pormenorizada esta relación, tendríamos que conceptualizar la conexión entre el consumo de drogas y las variables objeto de estudio, aspecto poco claro y controvertido con el que contamos actualmente.

El objetivo de este trabajo es comprobar si existe relación entre algunas variables de personalidad (autocontrol, asertividad, estilo atribucional para los éxitos, estilo atribucional para los fracasos y autoeficacia) con el consumo de drogas (cantidad y cronicidad de alcohol, hachís, cocaína y heroína).

Se espera encontrar que el autocontrol, la asertividad, la autoeficacia, el estilo atribucional para los éxitos, el estilo atribucional para los fracasos se relacionen con el consumo de drogas.

\section{3.- MÉTODO.}

\section{1- Sujetos.Sujetos}

Participaron en este estudio 53 sujetos, 19 mujeres y 34 hombres, con una edad media de 27.42 años, desviación típica de 4.49 y rango de 21-35 años. Los sujetos que componen la muestra son consumidores de drogas, las tablas 1 y 2 nos indican el perfil del consumo de la muestra de estudio (un $83 \%$ consume alcohol, un $38 \%$ hachis, un $42 \%$ cocaína y un $49 \%$ heroína). El nivel educativo de los sujetos comprende desde estudios primarios hasta licenciados. Específicamente, el $5.7 \%$ son licenciados, el $13.2 \%$ están realizando su licenciatura, el $9.4 \%$ son diplomados, el $5.7 \%$ están realizando su diplomatura, el 22.6\% tiene un nivel de estudios secundarios (BUP o FPII) y el $43.4 \%$ tiene un nivel de estudios primarios (EGB o 
FPI). Con respecto a su situación laboral el 56.6\% no trabajan mientras que el $43.4 \%$ si trabajan. Los sujetos fueron reclutados en lugares de encuentro de jóvenes (gimnasios, asociaciones, bares y centros de estudios y trabajo). El criterio de inclusión fue según respondieran a la Entrevista de Investigación acerca del Comportamiento Adictivo- Cuestionario de Investigación acerca del Comportamiento Adictivo (E.I.C.A), es decir, se incluyeron aquellos sujetos que contestaban que consumían a diarío al menos dos de las cuatro de las siguientes drogas: alcohol, hachís, cocaína y heroína y que llevaban consumiendo un tiempo mínimo de un año. Los sujetos completaban de forma voluntaria los cuestionarios, inventarios y escalas que a continuación se describen. Además se les motivó entregándoles un informe de su perfil de personalidad. También se les pidió información acerca de la edad (años), sexo (varón o mujer), nivel educativo (años de escolarización de acuerdo a una escala de 1 a 6) y situación laboral.

\section{2.- Medidas.}

Las medidas que se utilizaron en este estudio fueron:

3.2.1.- Cuestionario de Estilo Atribucional de Peterson et al. (1982) (A.S.Q). Este cuestionario mide las explicaciones dadas por los sujetos ante acontecimientos de éxito y fracaso. (A.S.EX)(A.S.FRA). El cuestionario consta de 12 situaciones, referidas 6 a acontecimientos de éxito y 6 a acontecimientos de fracaso. El sujeto puntúa de 1 a 7 la causa de estos acontecimientos en tres dimensiones: externa-interna, inestable-estable y específica-global, teniendo en cuenta que el 1 hace referencia a atribuciones externas, inestables y específicas y el 7 a atribuciones internas, estables y globales. Fue traducido y adaptado a la población española por Segura (1983). Peterson et al. (1982) obtuvieron una consistencia interna, con el coeficiente alfa de Cronbach de 0.75 y 0.72 para acontecimientos de éxito y fracaso, respectivamente, y una fiabilidad con el método test-retest para cada una de las dimensiones de éxito de 0.70 y de fracaso de 0.64. Con respecto al criterio de validación, estos autores hallaron una correlación de 0.19 y 0.41 entre las explicaciones espontáneas con las dimensiones del cuestionario en estudiantes, mientras Peterson y Seligman (1984) hallaron un coeficiente de correlación de $0.52,0.58$ y 0.52 para las explicaciones internas, estables y globales, analizando el grado de relación entre estilo atribucional y depresión en 100 estudios. Seligman et al. (1979) encontraron que un estilo atribucional interno, estable y global para los fracasos se asociaba con síntomas depresivos y Peterson et al. (1982) que los sujetos normales (no depresivos) tienden a hacer atribuciones internas, estables y globales de los éxitos. En esta misma línea, estos mismos autores, observaron que un estilo atri-

\begin{tabular}{|c|c|c|c|c|c|}
\hline DROGAS & UNIDADES DE CONSUMO & N DE SUJETOS QUE CONSUMEN & RANGO & Media & Desv. típ \\
\hline ALCOHOL & $\begin{array}{l}4-30 \\
48-96 \\
120-360\end{array}$ & $\begin{array}{l}20 \\
17 \\
16\end{array}$ & $4-360$ & 88.70 & 86.10 \\
\hline HACHIS & $\begin{array}{l}1-12 \\
24-72 \\
92-300\end{array}$ & $\begin{array}{l}7 \\
7 \\
7\end{array}$ & $0-300$ & 30.08 & 63.88 \\
\hline COCAÍNA & $\begin{array}{l}1 \\
8\end{array}$ & $\begin{array}{l}1 \\
1\end{array}$ & $0-8$ & 0.17 & 1.10 \\
\hline HEROÍNA & 0.20 & 1 & $0-0.20$ & 0.004 & 0.027 \\
\hline
\end{tabular}

\begin{tabular}{|c|c|c|c|c|c|}
\hline DROGAS & AÑOS DE CONSUMO & N DE SUJETOS QUE CONSUMEN & RANGO & Media & Desv. típ \\
\hline ALCOHOL & $\begin{array}{l}3-9 \\
10-19 \\
20-28\end{array}$ & $\begin{array}{l}21 \\
26 \\
6\end{array}$ & $3-28$ & 11.53 & 5.4 \\
\hline HACHÍS & $\begin{array}{l}1-3 \\
4-10 \\
11-17\end{array}$ & $\begin{array}{l}1 \\
10 \\
10\end{array}$ & $0-17$ & 3.9 & 5.55 \\
\hline COCAIINA & $\begin{array}{l}1-8 \\
9-11\end{array}$ & $\begin{array}{l}1 \\
1\end{array}$ & $0-11$ & 0.36 & 1.85 \\
\hline HEROIINA & 3 & 1 & $0-3$ & 0.06 & 0.41 \\
\hline
\end{tabular}


bucional interno para los acontecimientos de fracaso, conlleva una pérdida de autoestima, que una atribución estable conduce a dificultades de adaptación y, por último, que una atribución global refleja la cronicidad del déficit.

3.2.2.- Inventario de Asertividad de Rathus (1973) (A.R). - Inventario de Asertividad (AR) de Rathus (1973).Este inventario está compuesto por 30 items, respecto a los cuáles el sujeto debe indicar el grado de acuerdo o desacuerdo, en una escala de -3 a 3, con que cada uno de los items describe su propia conducta. Se trata de que el sujeto diga con exactitud si la frase propuesta es muy característica de su conducta. La versión en castellano de esta escala se puede encontrar en Carrobles (1979). Nevid y Rathus (1979) encontraron una buena fiabilidad con el procedimiento test-retest, de 0.77, y con el método de las dos mitades, de 0.77. Carrasco (1983) destacan los principales elementos que entran a formar parte de la conducta asertiva, analizándolos dentro de esta escala, siendo las manifestaciones personales (los items 2, 5, 11, 12, $13,14,16,17,20,21,22,23,26,29$ y 30), la defensa de los derechos e intereses personales (items 5, 14, 15, 17, 20, 26 y 30), comunicación de sentimientos personales (items 5, 14, 15, 17, 20, 26, 30), evitación de enfrentamientos personales (items 4, 7, 15, 19, 23, 24 y 26), manifestación pública de opiniones personales (items 7, 13, 16, 18, 23 y 29), espontaneidad (items $10,21,22,29$ y 30) y confrontación $(6,7,8,13,19,22$, 27 y 28$)$.

3.2.3.- Cuestionario de Autocontrol de Rosenbaum (1980)- Cuestionario de Autocontrol (AC) de Rosenbaum (1980) (AC). Evalúa la "competencia aprendida" o habilidades para controlar los efectos interfirientes de los eventos internos (tales como emociones y dolor o pensamientos no deseados) que afectan a la ejecución de la conducta. Consta de 36 items, respecto a los cuales el sujeto debe indicar el grado en que le describen o caracterizan, de acuerdo con una escala de -3 a 3. Traducido y adaptado por Capafóns y Barreto (1989). Capafóns (1986) revisa los estudios de fiabilidad y validez del cuestionario y encuentra que se han realizado dos, uno por el propio Rosenbaum (1980), que obtiene un coeficiente de fiabilidad de 0.86 por el método test-retest, y otro por Saéz et al. (1987) en el que el coeficiente de fiabilidad alcanza un valor de 0.82 , también con el método mencionado en el primer estudio. Entre los resultados de validez encontrados, dicho autor destaca una correlación negativa de -0.40 con el Cuestionario de Locus de Control de Rotter (1966), de -0.55 con la Escala de Ansiedad de Rasgo (STAI) de Spielberger et al. (1970) y de -0.56 con la Escala de Ansiedad Manifiesta (MAS) de Taylor (1953). Capafóns (1986) señala que este cuestionario es el único instrumento que evalúa los recursos psicológicos eficaces para manejar situaciones aversivas, desde la perspectiva del autocontrol. Además, se muestra como un buen predictor de éxito terapéutico (reducción de peso) y podría ser útil para establecer criterios de selección de terapias.

3.2.4.- Escala de Autoeficacia de Sherer et al. (1982).2.2.11.- Escala de Autoeficacia de Sherer y Madduxx (1982) (A.E). Esta escala fue desarrollada para analizar las creencias que tienen los sujetos acerca de sus habilidades para manejar determinadas situaciones. Fue traducida por Godoy (1990). La escala está compuesta de 30 ítems que miden la autoeficacia total. Los 30 items están divididos en 2 subescalas. En la subescala de autoeficacia general, el sujeto describe cómo maneja determinadas situaciones relacionadas con tareas personales y en la subescala de autoeficacia social, el sujeto describe como maneja determinadas situaciones sociales. En ambas escalas el sujeto debe indicar el grado de acuerdo de la frase que le describe o caracteriza en una escala de 1 a 5. La escala también incluye 7 ítems filler. En este estudio hemos utilizado la puntuación total (A.E). Sherer et al. (1982) señalan una consistencia interna, con el coeficiente alpha de Cronbach de 0.86 para la subescala de autoeficacia general y de 0.71 para la subescala de autoeficacia social y Woodruff y Cashman (1993) obtienen un coeficiente alpha de Cronbach de 0.84 para la subescala de autoeficacia general y de 0.69 para la subescala de autoeficacia social. Con respecto al criterio de validación, Sherer et al. (1982) señalan una validez convergente con otras medidas de características de personalidad (Locus de Control, Control Personal, Fuerza del Ego, Competencia Interpersonal) relacionadas con la eficacia general sin ser sinónimas. Esta escala se ha utilizado para analizar la relación entre el esfuerzo y persistencia de los comportamientos elegidos y otras conductas relacionadas con la salud, sobre todo en lo que respecta a la adherencia a tratamientos y al pronóstico en rehabilitación de enfermedades crónicas. Una alta puntuación en esta escala indica niveles elevados de autoeficacia, mientras que una baja puntuación, es indicativa de ausencia de autoeficacia.

3.2.5.- Entrevista de Investigación acerca del Comportamiento Adictivo- Cuestionario de Investigación acerca del Comportamiento Adictivo (E.I.C.A). Fue desarrollado para detectar el consumo de alcohol y drogas en el momento de la evaluación, versa sobre el comportamiento adictivo, particularmente del consumo de alcohol, hachís, cocaína y heroína. Hace referencia a la frecuencia de consumo de estas sustancias, cantidad de consumo y edad de inicio de las mismas. Consta de 7 items que se dividen en tres grupos de preguntas, un grupo hace referencia a la frecuencia de consumo en el último mes que puede ser a diario, entre una y tres veces a la semana, una vez a la semana, entre una y tres veces al mes y una vez al mes. Un segundo grupo también hace referen- 
cia a los gramos, mililitros o unidades. Finalmente, un tercer grupo hace referencia al tiempo de consumo en años desde que se inició el consumo. Con este último grupo calculamos la cronicidad del consumo de drogas que se puntúa en años y se valora el tiempo de consumo desde la edad de inicio del consumo hasta el momento de la evaluación. Con el primer y segundo grupo de preguntas calculamos la cantidad de drogas consumidas en el último mes, el primer grupo es indicativo de si el consumo es diario, semanal o mensual, se puntúa desde 1 hasta 8 y oscila desde nunca hasta a diario; el segundo grupo es indicativo del número de copas, cigarrillos o gramos que se consumen, se puntúa teniendo en cuenta que un whisky equivale a 1 unidad y un vino o una cerveza equivale a 0.5 , el hachís se puntúa en número de cigarrillos y la cocaína y heroína en gramos al mes. A partir de este cuestionario se obtienen 8 medidas que informan sobre la frecuncia de uso y unidades de consumo (cantidad de alcohol, hachís, cocaína y heroína.), así como los años transcurridos desde que se inició el consumo (cronicidad de alcohol, hachís, cocaína y heroína). Finalmente se obtienen 2 puntuaciones estandarizadas (tipificadas) que definen el consumo de drogas, una es la cantidad de consumo de drogas y otra es la cronicidad de dicho consumo.

\section{3.- Variables y Análisis estadísticos:}

\subsection{1.- Variables Dependientes:}

- Puntuación estandarizada de la cantidad de alcohol, hachís, cocaína y heroína derivada de la Entrevista de Investigación acerca del Comportamiento Adictivo- Cuestionario de Investigación acerca del Comportamiento Adictivo E.I.C.A (Z-CANTIDAD DE DROGA).

- Puntuación estandarizada de la cronicidad del consumo de alcohol, hachís, cocaína y heroína derivada de la Entrevista de Investigación acerca del Comportamiento Adictivo- Cuestionario de Investigación acerca del Comportamiento Adictivo (E.I.C.A) (Z-CRONICIDAD DE DROGA).

\subsection{2.- Variables Independientes:}

- Puntuación del estilo atribucional para los éxitos derivada del Cuestionario de Estilo Atribucional de Peterson et al. (1982) (A.S.EX).

- Puntuación del estilo atribucional para los fracasos derivada del Cuestionario de Estilo Atribucional de Peterson et al. (1982) (A.S.FRA).

- Puntuación de asertividad derivada del Inventario de Asertividad de Rathus (1973) (A.R).

- Puntuación de autocontrol derivado del Cuestionario de Autocontrol de Rosenbaum (1980)- Cuestionario de Autocontrol (AC) de Rosenbaum (1980) (A.C).
- Puntuación de autoeficacia derivada de la Escala de Autoeficacia de Sherer et al. (1982).2.2.11.Escala de Autoeficacia de Sherer y Madduxx (1982) (A.E).

Para contrastar la principal hipótesis del estudio, se llevaron a cabo dos análisis de regresión lineal múltiple, con el objetivo de determinar la relación entre estilo atribucional para los éxitos y estilo atribucional para los fracasos, asertividad, autocontrol, autoeficacia y consumo de drogas (cantidad y cronicidad de alcohol, hachís, cocaína y heroína consumidas).

\section{4.- Procedimiento}

Se realizó una entrevista inicial individualizada, en la que se da información de la investigación y se pregunta al sujeto si quiere participar. En caso positivo, éste comienza realizando los cuestionarios de información acerca del nivel cultural y categoría profesional y de la entrevista de investigación acerca del comportamiento adictivo. Así, se les motiva diciéndoles que, al finalizar el cumplimiento de los cuestionarios, inventario y escala, se entregará un informe con los resultados de su perfil de personalidad. En segundo lugar, se comienza, de manera individualizada, a administrar de forma contrabalanceada los diferentes cuestionarios, inventario y escala. Las normas de aplicación son las estandarizadas de cada prueba, es decir, el sujeto se sienta en la mesa frente al experimentador y éste le entrega el cuadernillo de los cuestionarios y un bolígrafo, diciéndole que escriba su nombre y lea atentamente las instrucciones. Una vez que el experimentador se asegura que el sujeto ha entendido las instrucciones y la lógica de los items, éste último procede a responder.

\section{4.- RESULTADOS}

\section{Resultados}

Se realizaron dos análisis de regresión lineal múltiple para cada una de las variables que definen el consumo de drogas: cantidad de droga consumida (alcohol, hachís, cocaína y heroína) y cronicidad del consumo de drogas (años de consumo de alcohol, hachís, cocaína y heroína). En los dos análisis de regresión lineal múltiple las variables independientes son las mismas variables de personalidad (estilo atribucional para los éxitos y estilo atribucional para los fracasos, asertividad, autocontrol y autoeficacia).

El análisis de varianza debido a la primera regresión (cantidad de drogas consumidas) refleja la significación estadística global de la relación conjunta de la cantidad de drogas consumidas con las variables independientes (estilo atribucional para los éxitos y estilo 
atribucional para los fracasos, asertividad, autocontrol y autoeficacia). Los resultados indican que el autocontrol y la asertividad se relacionan con la cantidad de drogas consumidas, $F=4.673,4 / 52 \mathrm{gl}, \mathrm{p}<.001$; explicando el $53 \%$ de la varianza total. No se encuentran diferencias estadísticamente significativas para la relación entre estilo atribucional para los éxitos y fracasos, autoeficacia y la cantidad de drogas consumidas.

El análisis de varianza debido a la segunda regresión (cronicidad del consumo de drogas) refleja la significación estadística global de la relación conjunta de la cronicidad del consumo de drogas) con las variables independientes (estilo atribucional para los éxitos y estilo atribucional para los fracasos, asertividad, autocontrol y autoeficacia). Los resultados indican que el autocontrol y la asertividad también están relacionadas con la cronicidad del consumo de drogas, $F=$ 4.235, 4/52 gl, p<.005; explicando el $51 \%$ de la varianza total. Tampoco se encuentran diferencias estadísticamente significativas para la relación entre estilo atribucional para los éxitos y fracasos, autoeficacia y cronicidad del consumo de drogas.

Los resultados de significación estadística de cada coeficiente nos indican, por un lado, que el autocontrol aparece inversamente relacionado con la cantidad de drogas consumidas ( $B=-0.522, t=-3.837, p<.000)$ y con la cronicidad del consumo de drogas $(B=-0.504$, $t=-3.656, p<.001)$. Por otro lado, la asertividad aparece directamente relacionada con la cantidad de drogas consumidas $(B=0.405, t=2.77, p<.008)$ y con la croni- cidad del consumo de drogas $(B=0.402, t=2.710$, $\mathrm{p}<$.009).

En la tabla 3 aparecen las medias, desviaciones tipicas y rango de cada una de las variables de personalidad analizadas; en la tabla 4 observamos los coeficentes de determinación y el modelo de ecuaciones de regresión lineal múltiple que cuantifica la relación, especificando el peso de cada una de las variables independientes en la fórmula; en la tabla 5 se aprecian las variables que se relacionan con el consumo de drogas (cantidad y cronicidad), los coeficientes estandarizados (divididos por su error) (B) y la prueba de significación (t). Los coeficientes estandarizados obtenidos de cada variable indican que la probabilidad de consumir drogas, con respecto al autocontrol y asertividad, representan para el autocontrol el decremento y para la asertividad el incremento de consumir drogas. Por tanto, un drogodependiente con una puntuación de 96 en autocontrol, presenta 0.522 y 0.504 veces menos probabilidad de consumir drogas y con una puntuación de 49 en asertividad, presenta 0.405 y 0.402 veces más probabilidad de consumir drogas.

\section{5.- DISCUSIÓNY CONCLUSIONES.}

Los resultados obtenidos resaltan que el consumo de drogas, definido como la cantidad y cronicidad del consumo de drogas (alcohol, hachís, cocaína y heroí-

\begin{tabular}{|c|c|c|c|c|}
\hline \multicolumn{5}{|c|}{$\begin{array}{l}\text { Tabla 3: Medias, desviaciones típicas y } \\
\text { rango de las variables de personalidad }\end{array}$} \\
\hline Variables & Media & Desviación Típica & Rango & \\
\hline & & & Mínimo & Máximo \\
\hline A.C & 34.55 & 22.51 & -36 & 96 \\
\hline A.R & 6.96 & 16.73 & -41 & 41 \\
\hline A.E & 84.51 & 12.83 & 44 & 112 \\
\hline A.S.EX & 4.87 & 0.79 & 2.70 & 6.5 \\
\hline A.S.FRA & 3.85 & 0.82 & 1.72 & 6.67 \\
\hline
\end{tabular}

A.C= Autocontrol.

A.R=Asertividad.

A.E $=$ Autoeficacia.

A.S.EX= Estilo atribucional para los éxitos.

A.S.FRA = Estilo atribucional para los fracasos

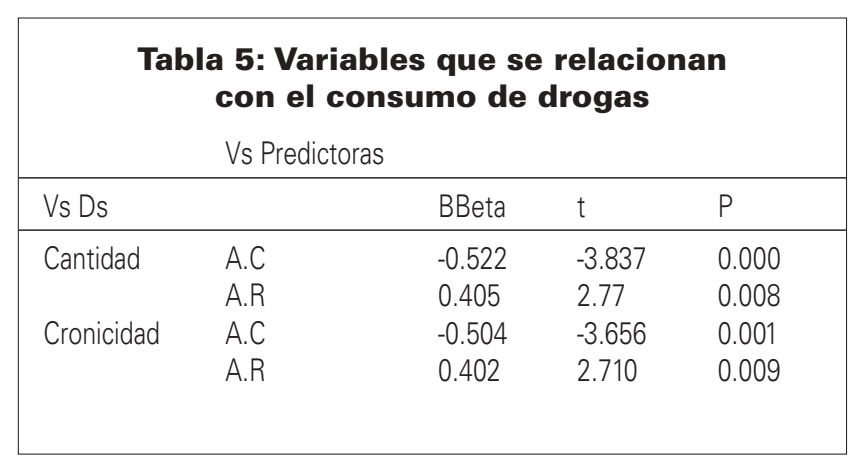

A.C $=$ Autocontrol

A. $R=$ Asertividad.

$\mathrm{B}=$ Coeficientes estandarizados.

\section{Tabla 4: Ecuaciones de regresión y coeficientes de determinación}

\begin{tabular}{|lll|} 
Modelos & R2 & Ecuaciones de regresión \\
\hline 1 & 0.53 & Cantidad de consumo de drogas $=0.674-0.522 \mathrm{AC}+0.405 \mathrm{AR}$ \\
\hline 2 & 0.51 & Cronicidad de las drogas consumidas $=0.671-0.504 \mathrm{AC}+0.402 \mathrm{AR}$ \\
\hline
\end{tabular}

\footnotetext{
R2= Coeficiente de determinación
} 
na) está relacionado de manera inversa con el autocontrol y de manera directa con la asertividad.

Por tanto, podemos concluir que el autocontrol se relaciona con el consumo de drogas de manara inversa, es decir, a medida que los niveles de autocontrol disminuyen, aumentan la cantidad y cronicidad de consumo de drogas, por lo que se deduce que si un sujeto presenta una puntuación baja en autocontrol la probabilidad de consumo de drogas aumenta. Observamos que esta variable explica una parte importante de la variabilidad, tanto en la cantidad de consumo como en la cronicidad del mismo. Cabe deducir, en términos generales, que se confirman una vez más las tendencias que se hallaron en los estudios previos, en esta ocasión con una muestra andaluza de consumidores de drogas. Nuestros resultados son consonantes con los obtenidos por D’Elio et al. (1996); Santacreu y Froján (1992) y Wills (1994) aunque estos autores analizan dicha variable con poblaciones. Resaltamos también que Sterling et al. (1996); Wills (1994) obtienen coeficientes de determinación inferiores a los que nosotros obtenemos y finalmente, D'Elio et al. (1996) hallan un coeficiente (B) también inferior a los nuestros.

El segundo efecto significativo, nada despreciable lo encontramos en la asertividad que también se relaciona, en sentido directo, con el consumo de drogas (tanto la cantidad de consumo como la cronicidad del mismo). Encontramos nuevamente cierta consonancia con los resultados obtenidos por la literatura revisada; específicamente, Wills et al. (1989) obtienen que la asertividad está directamente relacionada con el consumo de alcohol y Baldwin et al. (1993) afirman que las expectativas de sentirse más asertivos bajo los efectos del alcohol se relacionan positivamente con la cantidad de alcohol.

Otras variables analizadas han sido el estilo atribucional y la autoeficacia que no contribuyen, según nuestros datos, a predecir la cantidad y cronicidad de consumo de drogas analizadas.

Con respecto al estilo atribucional, no encontramos ningún efecto entre dicha variable y el consumo de drogas, tal y como se resalta en la literatura revisada, es decir, Dowd et al. (1986) y Echeburúa y Elizondo (1988) hallan que las atribuciones estables y globales para los éxitos estaban asociadas con el abandono en el tratamiento de los alcohólicos y que el estilo atribucional de los alcohólicos para los éxitos es externo e inestable y para los fracasos es interno y global. Ante los datos, aparentemente diferentes, dado que en nuestro estudio no encontramos efecto entre el estilo atribucional y el consumo de drogas, considérese que en las muestras que componen los estudios revisados son únicamente de alcohólicos que están en tratamiento. Esto nos permite plantearnos la necesidad de realizar, en este tipo de investigaciones, los análisis adecuados que permitan tener en cuenta el nivel de severidad en el consumo de drogas y la complejidad de las variables analizadas. Desde la literatura revisada y desde nuestros propios resultados entendemos que en función de las variables estudiadas, del nivel de profundización de la severidad del consumo de alcohol y drogas, de la muestra utilizada (inicio de consumo, tratamiento o recaída) las conclusiones a las que se pueden llegar resultan dificilmente generalizables.

Otra variable estudiada, la autoeficacia, no contribuye según nuestros datos, a establecer relaciones con el consumo de drogas. Estos resultados van en contra de nuestra hipótesis inicial, ya que esperábamos que dicha variable pudiera predecir el consumo de drogas, aunque destacamos que van en la línea de los obtenidos por otros autores, como Dowd et al. (1986), que afirman que no existen diferencias entre la autoeficacia y el consumo de alcohol. Quizás dicha variable está más relacionada con otras etapas de las drogodependencias, como puede ser el tratamiento o el mantenimiento de la abstinencia. Esta idea se halla en consonancia con los resultados obtenidos por Rist y Watzl (1983) quiénes afirman que las puntuaciones altas en autoeficacia están asociadas a la convicción por parte de los sujetos de sentirse capaces de mantener la abstinencia en el momento de iniciar el tratamiento; Hays y Ellickson (1990) encuentran que puntuaciones altas en autoeficacia están relacionadas con conductas de abstinencia; Baldwin et al. (1993) encuentran que los sujetos que puntúan alto en autoeficacia mantienen niveles bajos de consumo de alcohol y, finalmente, Sadowski et al. (1993) afirman que los sujetos que habían realizado tratamiento presentaban puntuaciones más altas en autoeficacia que aquellos que no habían realizado tratamiento.

A lo largo de este trabajo hemos expuesto que en la actualidad existen pocos estudios que relacionen, el consumo de drogas (cantidad y cronicidad de alcohol, hachís, cocaína y heroína) con variables de personalidad (autocontrol, asertividad, autoeficacia y estilo atribucional). Desde la evidencia que presentamos, pensamos en la conveniencia de incluir entrenamiento en autocontrol y asertividad como forma de prevención en el consumo posterior. Los sujetos consumen drogas en función de la interacción de diversos factores sociales, familiares, de aprendizaje y personales, pero donde también se tendría que incidir es en las variables que han resultado tener efecto. 
Abramson, L.Y., Seligman, M.E.P. y Teasdale, J. (1978). Learned helplessness in humans: Critique and reformulation. Journal of Abnormal Psychology, 87, 49-74.

Baldwin, A.R., Oei, T.P.S. y Young, R. (1993). To drink or not to drink: The differential role of alcohol expectancies and drinking refusal self-efficacy in quantity and frequency of alcohol consumption. Cognitive Therapy and Research, 17, 511-530.

Capafóns, A. (1986). Un cuestionario de autocontrol para niños y adolescentes: Primeros resultados. Análisis y Modificación de Conducta, 12, 31-32.

Capafóns, A. y Barreto, P. (1989). Competencia aprendida: Fiabilidad y validez de su medida, criticas y recomendaciones. Revista Española de Terapia del Comportamiento, 7, 19-39.

Carrasco, I. (1983). Enfoque cognitivo- conductual de los problemas de aserción. Revista Española de Terapia del Comportamiento, 1, 129-142.

Carrasco, I., Clemente, M. y LLavona, L. (1983). Análisis de componentes básicos del inventario de asertividad de Rathus. Revista Española de Terapia del Comportamiento, 1, 249-264.

Carrobles, J.A. (1979). Escala de asertividad de Rathus. En: P. Bartolome, J.A. Carrobles, M. Costa, T. Del Ser (Eds). La práctica de la terapia de conducta. Madrid: Promolibro. (pp. 189).

D’Elio, M.A., O’Brien, R.W., lannotti, R.J., Bush, P.J. y Galper, D.I (1996). Early adolescents' substance use and life stress: Concurrent and prospective relationships. Substance Use and Misuse, 31, 873-894.

Dowd, E.T., Lawson, G.W. y Petosa, R. (1986). Attributional styles of alcoholics. The International Journal of the Addictions, 21, 589-593.

Echeburúa, E. y Elizondo, M. (1988). Estilo atribucional y locus de control en una muestra de alcohòlicos anònimos. Revista Vasca de Psicologia, 1, 71-78.

Godoy, J.F. (1990). Escala de Autoeficacia. Versión experimental, no publicada.

Hays, R.D. y Ellickon, P.L. (1990). How generalizable are adolescents beliefs about pro-drug pressures and resistance self-efficacy?. Journal of Applied Social Psychology, 20, 321-340.

Kahler, C.W., Epstein, E.E., McCrady, B.S. (1995). Loss of control and inability to abstain: The measurement of and the relationship between two constructs in male alcoholics. Addiction, 90, 1025-1036.

Nevid, J.S. y Rathus, S.A. (1979). Factor analysis of the Rathus assertiveness schedule with a college population. Journal of Behavior Therapy and Experimental Psychiatry, 10, 21-24.

Peterson, C. y Seligman, M. (1984). Causal explanations as risk factor for depression: Theory and evidence. Psychological Review, 91, 147-174.

Peterson, C., Semmel, A., Von Baeyer, C., Abramson, L.Y., Metalsky, L.I., y Seligman, M.E.P. (1982). The attributio nal style questionnaiere. Cognitive Therapy Research, 6, 287-300

Rathus, S.A. (1973). Escala de asertividad. En P. Bartolomé, J.A. Carrobles, M. Costa, T. Del Ser (Eds.).(1979). La práctica de la terapia de conducta. Madrid: Pablo del Rio. (apéndice).

Reich, J.W. y Gutiérres, S.E. (1987). Life event and treatment attributions in drug abuse and rehabilitation. American Journal Drug Alcohol Abuse, 13, 73-94.

Rist, F. y Watzl, H. (1983). Self assessement of relapse risk and assertiveness in relation to treatment outcome of female alcoholics. Addictive Behaviors, 8, 121-127.

Rizley, R. (1978). Depression and causal attributions for sucess and failure. Journal Abnormal Psychology, 87, 32-48.

Room, R. (1989). Drugs, consciousness and self-control: Popular and medical conceptions. Special Issue: Psychiatry and the addictions. International Review of Psychiatry, 1, 63-70.

Rosenbaum, M. (1980). Cuestionario de autocontrol. En A. Capafóns y P. Barreto (1989) (traducción y adaptación). Competencia aprendida: Fiabilidad y validez de su medida, criticas y recomendaciones. Revista Española de Terapia del Comportamiento, 1, 19-39.

Rotter, J. (1966). Generalized expetancies for internal vesus external control reinforcement. Psychological Monographs. 80, volumen completo.

Sadowski, C.J., Long, C.L. y Jenkins, L.R. (1993). Does substance abuse treatment have self-schematic effects?. The Journal of Psychology, 127, 323-327.

Saéz, A., Aliaga, F y Capafons, A. (1987). Citado en A. Capafóns y P. Barreto (1989). Competencia aprendida: Fiabilidad y validez de su medida, críticas y recomendaciones. Revista Española de Terapia del Comportamiento, 1, 19-39.

Santacreu, J. y Froján, M.X. (1992). El papel del autocontrol en el proceso de génesis de las drogodependencias II. Revista Española de Drogodependencias, 17, 253268.

Segura, M. (1983). Cuestionario de Estilo Atribucional. Segundas Jornadas de Modificación de Conducta. Centro de Investigación y Terapia de Conducta. Madrid.

Seligman, M.E.P., Abramson, L., Semmel, A. y Von Baeyer, C. (1979). Depressive attributional style. Journal of Abnormal Psychology, 88, 242-247.

Sherer, M., Maddux, J.E., Mercandante, B., Prentice-Dunn, S., Jacobs, B. y Rogers, R.W. (1982). The Self-efficacy Scale: Construction and validation. Psychological Reports. 51, 663-671.

Shiffman, S. y Wills, T.A. (1985). Coping and cesation of subtance use. En S. Shiffman y T.A. Wills. (Eds.). Coping and substance use. New York: Academic Press. (pp. 221-240).

Spielberger, C.D., Gorsuch, R.L. y Lushene, R.E. (1970). Manual for the state-trait anxiety inventory. Palo 
Alto, California: Consulting Psychologicist Press. (Adaptación española: TEA, ediciones, 1982).

Sterling, R.C., Gotteheil, E., Weinstein, S.P., Lundy, A. y Serota, R.D. (1996). Learned helplessness and cocaine dependence: An investigation. Journal of Addictive Diseases, 15, 13-24.

Taylor, J. (1953). Escala de ansiedad manifiesta. Citado en A. Capafóns y P. Barreto (1989). Competencia aprendida: Fiabilidad y validez de su medida, criticas y recomendaciones. Revista Española de Terapia del Comportamiento, 7, 19-39.

Van Hasselt, V.B., Null, J.A., Kempton, T. y Bukstein, O.G. (1993). Social skills and depression in adolescent subtance abusers. Addictive Behaviors, 18, 9-18.
Wills, T.A. (1994). Self-esteem and perceived control in adolescent substance use: Comparative tests in concurrent and prospective analyses. Psychology of Addictive Behaviors, 8, 233-234.

Wills, T.A., Baker, E. y Botvin, G.J. (1989). Dimensions of assertiveness: Differential relationships to substance use in early adolescence. Journal of Consulting and Clinical Psychology, 57, 473-478.

Woodruff, S.L. y Cashman, J.F. (1993). Task, domain and general efficacy: A reexamination of the Self-efficacy Scale. Psychological Reports. 72: 423-432. 